ARTÍCULO ORIGINAL

Versión impresa ISSN 1666-938X

Versión digital ISSN 2362-5589

\title{
Especies de garrapatas (Acari: Ixodidae) parásitas de perros (Canis familiaris) en zonas urbanas del Gran Mendoza, Argentina
}

\author{
Fantozzi $\mathrm{MC}^{1^{*}}$, Linares $\mathrm{MC}^{2}$, Cuervo $\mathrm{PF}^{1}$, Romoli $\mathrm{A}^{3}$, Vittaz $\mathrm{D}^{3}$ y Mera y Sierra $\mathrm{R}^{3}$ \\ ${ }^{1}$ Laboratorio de Ecología de Enfermedades, Instituto de Ciencias Veterinarias del Litoral, \\ Universidad Nacional del Litoral / Consejo Nacional de Investigaciones Científicas y Técnicas, \\ Esperanza, Santa Fe, Argentina. \\ 2 Instituto de Biología y Medicina Experimental/Consejo Nacional de Investigaciones Científicas y \\ Técnicas, Ciudad Autónoma de Buenos Aires, Argentina. \\ ${ }^{3}$ Centro de Investigación en Parasitología Regional (CIPAR), Facultad de Ciencias Veterinarias y \\ Ambientales. Universidad Juan Agustín Maza, Mendoza, Argentina. \\ * Correspondencia: María Cecilia Fantozzi, LEcEn,ICIVET Litoral (UNL-CONICET), RP Kreder 2805, \\ 3080 Esperanza, Santa Fe, Argentina. \\ E-mail: cfantozzi@fcv.unl.edu.ar
}

Recibido: 19 Marzo 2018. Aceptado: 12 Junio 2018. Disponible en línea: 21 Junio 2018

Editor: S. Nava

RESUMEN. El conocimiento de las especies de ectoparásitos presentes en los animales de compañía tiene implicancias tanto en medicina veterinaria como en salud humana, ya que varios agentes tienen la potencialidad de transmitirse del animal al humano y viceversa. Se revisaron 466 garrapatas remitidas al Centro de Investigación en Parasitología Regional (CIPaR) durante los años 2010 y 2013, recolectadas en 217 perros de zonas urbanas de la provincia de Mendoza. El 83,5\% ( $n=385$ ) fueron identificadas como Rhipicephalus sanguineus sensu lato (84 machos y 199 hembras - relación 1:2,37 -, 65 ninfas y 37 larvas), mientras que el 16,5\% ( $n=76$ ) fue identificado como Amblyomma tigrinum (10 machos y 61 hembras - relación 1:6,1 -, y cinco ejemplares adultos en mal estado, imposibilitando su sexado). Adicionalmente, cinco ejemplares adultos sólo se identificaron como pertenecientes al género Amblyomma, ya que por encontrarse ingurgitados no se pudo realizar la determinación de especie. Éste registro es un importante aporte al conocimiento de las especies de garrapatas presentes en perros (Canis familiaris) en la provincia de Mendoza.

SUMMARY. Ticks species (Acari: Ixodidae) parasites of dogs (Canis familiaris) in urban areas from Mendoza province, Argentina. Knowledge of the ectoparasitic agents of pets has implications both in veterinary medicine and public health since several agents have the potential to spread from animal to human and vice versa. Ticks sent to the Centro de Investigación en Parasitología Regional (CIPaR) during 2010 to 2013 were analysed. 466 adult ticks were collected from 217 dog in urban areas from Mendoza province, Argentina. Of these, $83.5 \%(\mathrm{n}=385)$ specimens were identified as Rhipicephalus sanguineus s.l. (84 male and 199 female - ratio 1:2.37 -, 65 nymphs and 37 larvae), while 16.5\% ( $n=76$ ) were identified as Amblyomma tigrinum (10 males and 61 females - ratio 1:6.1 -, and five adults in bad condition, which precluded their sexing). In addition, five adult specimens, identified as Amblyomma, were not identified to the species level since they were fully engorged. This report is an important contribution to the knowledge of the species of ticks in dogs (Canis familiaris) in the province of Mendoza.

Palabras clave: garrapatas, Rhipicephalus sanguineus s.l., Amblyomma tigrinum, Mendoza, perros

Key words: ticks, Rhipicephalus sanguineus s.I., Amblyomma tigrinum, Mendoza, dogs

\section{Introducción}

Las garrapatas duras son parásitos hematófagos que presentan una amplia diversidad de hospedadores (Nava et al., 2017), ocasionando en algunos casos problemas sanitarios para los mismos (Guglielmone et al., 2004).
Estos artrópodos están considerados entre los más importantes vectores de enfermedades infecciosas, ya que poseen la capacidad de transmitir microorganismos patógenos como virus, bacterias y protozoos; que pueden 
afectar gravemente al hospedador, e incluso ocasionar su muerte (Parola et al., 2013; Nava et al., 2017). Esta situación conlleva un considerable impacto económico y sanitario en producción animal, salud animal y salud pública (Guglielmone et al., 2004; Nava et al., 2017). Dado el estrecho contacto que actualmente se da entre los perros y las personas, éstos cumplen un rol importante en el mantenimiento de diversas zoonosis (DantasTorres, 2010). Describir las asociaciones garrapatasperros permite realizar inferencias epidemiológicas sobre las enfermedades transmitidas por vectores a los perros y diseñar estrategias de control (Debárbora et al., 2011). Actualmente las enfermedades transmitidas por garrapatas representan un problema, emergente o reemergente según los an-tecedentes de cada sitio (Eiras et al., 2007, 2008; Dantas-Torres, 2010; Parola et al., 2013; Monje et al., 2016; Cicuttin et al., 2017a).

En Argentina, las garrapatas que afectan a caninos domésticos corresponden en su mayoría al complejo Rhipicephalus sanguineus y a distintas especies de Amblyomma. En América, Rh. sanguineus sensu lato está conformada por al menos dos linajes con distinta distribución biogeográfica y posiblemente distinta competencia vectorial (Moraes-Filho et al., 2011). El linaje tropical se ubica en localidades tropicales y subtropicales, incluyendo el norte de Argentina, y se encuentra relacionado filogenéticamente con garrapatas del complejo $R h$. sanguineus de África; mientras que el linaje templado se distribuye en localidades templa-das de Argentina, Chile y Uruguay, y se relaciona con garrapatas del complejo Rh. sanguineus del oeste de Europa (Nava et al., 2012). Si bien esta garrapata ha sido encontrada en otras especies de mamíferos (incluyendo el hombre), el perro es el principal hospedador de todos sus estadíos (Walker et al., 2000). La relevancia médico-veterinaria de $R h$. sanguineus s.l. se debe a su rol como vector de agentes patógenos como Ehrlichia canis, Anaplasma platys, Babesia canis vogeli, Babesia gibsoni, Hepatozoon canis, Rickettsia rickettsii, Rickettsia conorii o Richettsia massiliae, muchos de los cuales también pueden afectar a humanos (Walker et al., 2000). En un estudio reciente en la provincia de Mendoza, se reportó la presencia de ejemplares de $R h$. sanguineus (linaje templado) infectados con Rickettsia massiliae (Monje et al., 2016).

En cuanto al género Amblyomma, se han reportado al menos 6 especies que parasitan perros en el cono sur de América (Amblyomma varium, Amblyomma aureolatum, Amblyomma hadanii, Amblyomma ovale, Amblyomma tigrinum y Amblyomma triste) (Nava et al., 2017). Las especies de este género, además de ser parásitos frecuentes de animales domésticos, ocasionan la mayoría de las infecciones por garrapatas en seres humanos (Nava et al., 2017); al tiempo que han sido identificadas como responsables de la transmisión al hombre de importantes microorganismos patógenos (Labruna et al., 2007; Venzal et al., 2012; Parola et al., 2013; Cicuttin, 2014; Romer et al., 2014).
El objetivo del presente trabajo fue determinar las especies de garrapatas que parasitan a caninos domésticos de diferentes departamentos que involucran zonas urbanas del Gran Mendoza, Argentina.

\section{Materiales y Métodos}

Se revisaron las garrapatas remitidas al Centro de Investigación en Parasitología Regional (CIPaR) de la Facultad de Ciencias Veterinarias y Ambientales (Universidad Juan A. Maza), en un periodo compren-dido entre 2010-2013. Las mismas provenían de los principales ejidos urbanos de los departamentos que conforman el área conocida como Gran Mendoza: Godoy Cruz, Luján, Guaymallén, Lavalle, Capital y Las Heras (Fig. 1). El clima del Gran Mendoza está caracterizado por ser templado, variando de templado frío a templado cálido, según se avanza de sur a norte (Informe I.N.V., 2016). Las muestras fueron recolec-tadas sobre perros en forma manual, conservadas en alcohol 96ㅇ. La identificación se realizó con lupa estereoscópica Labomed CZM6 (4X), siguiendo las claves taxonómicas de identificación de Nava et al., (2017).

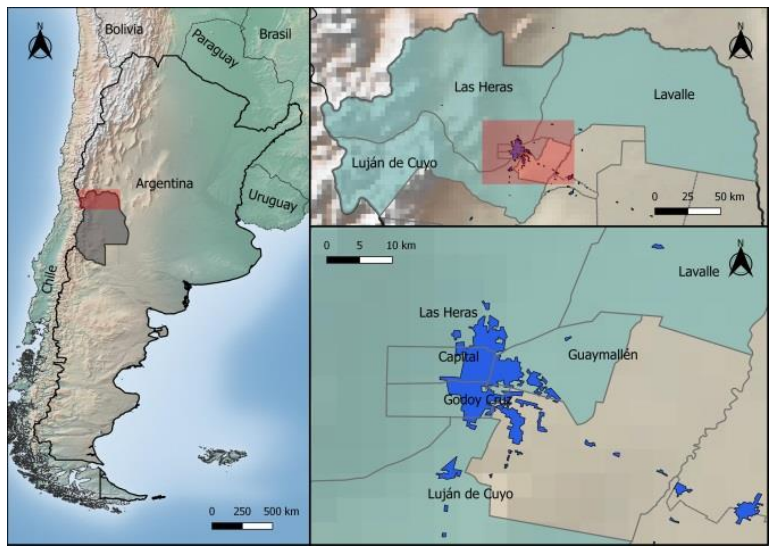

Figura 1. Localización geográfica de los departamentos de origen de las muestras remitidas. La coloración azul señala los ejidos urbanos en cada uno de ellos.

\section{Resultados}

Se recibieron 466 garrapatas colectadas sobre 217 caninos. El $83,5 \%(n=385)$ de las muestras fueron identificadas como $R h$. sanguineus s.l., correspondiendo a 84 machos y 199 hembras, en una relación 1:2,37, 65 ninfas y 37 larvas; mientras que el 16,5\% ( $n=76)$ fue identificado como A. tigrinum; 10 machos y 61 hembras, en una relación 1:6,1; además de cinco ejemplares adultos que se encontraban en mal estado, lo que imposibilitó su sexado. En el caso de otros cinco ejemplares adultos, no se pudo llegar al diagnóstico de especie por encontrarse ingurgitadas, por lo que sólo se identificaron como del género Amblyomma. 
En la Tabla 1 se observan las frecuencias de las dos especies de garrapatas registradas en este trabajo, según el departamento de origen. Cerca del $85 \%$ de las muestras $(n=390)$ fueron remitidas de los departamentos de Las Heras, Godoy Cruz y Luján de Cuyo, de las cuales el $85,4 \%$ fueron ejemplares de $R h$. sanguineus s.l., y $14,6 \%$ restante $A$. tigrinum.

Por tratarse de muestras recibidas de manera pasiva, no se cuenta con datos de prevalencia, carga parasitaria, o similares, que permitan inferir sobre características poblacionales.

Tabla 1. Especies de garrapatas asociadas a perros en ejidos urbanos de los departamentos que conforman el Gran Mendoza, Argentina.

\begin{tabular}{|l|c|c|c|}
\hline Departamento & $\begin{array}{c}\text { Amblyomma } \\
\text { tigrinum }\end{array}$ & $\begin{array}{c}\text { Riphicephalus } \\
\text { sanguineus s.I. }\end{array}$ & TOTAL \\
\hline Godoy Cruz & $6(5 \%)$ & $115(95 \%)$ & $121(26,2 \%)$ \\
\hline Luján de Cuyo & $34(25,4 \%)$ & $100(74,6 \%)$ & $134(29,1 \%)$ \\
\hline Guaymallén & $0(0 \%)$ & $48(100 \%)$ & $48(10,4 \%)$ \\
\hline Lavalle & $19(95 \%)$ & $1(5 \%)$ & $20(4,3 \%)$ \\
\hline Capital & $0(0 \%)$ & $3(100 \%)$ & $3(0,6 \%)$ \\
\hline Las Heras & $17(12,6 \%)$ & $118(87,4 \%)$ & $135(29,3 \%)$ \\
\hline TOTAL & $\mathbf{7 6 ( 1 6 , 5 \% )}$ & $\mathbf{3 8 5}(\mathbf{8 3}, \mathbf{5 \% )}$ & $\mathbf{4 6 1 ( 1 0 0 \% )}$ \\
\hline
\end{tabular}

\section{Discusión}

En el presente trabajo, un poco más del $80 \%$ de las garrapatas provenientes de caninos domésticos correspondieron a ejemplares de $R h$. sanguineus s. I., predominando en las muestras remitidas de todos los departamentos del Gran Mendoza (con excepción de Lavalle). Esta especie de garrapata intervendría en la transmisión de $H$. canis y $B$. canis vogeli en otras regiones del país, de acuerdo a lo propuesto por Eiras et al., (2007, 2008). Asimismo, cabe destacarse que Rh. Sanguineus s.l. ha sido señalada como un potencial transmisor de $R$. massiliae a humanos (Cicuttin, 2014), y que incluso se ha reportado su presencia con éste patógeno en la provincia de Mendoza (Monje et al., 2016). Por otro lado, como se mencionó previamente, en la ciudad de Mendoza se encontraría presente el linaje templado de $R h$. sanguineus s.l. el cual participaría de la transmisión de E. canis en la ciudad de Buenos Aires (Cicuttin et al., 2017a). Al respecto, es relevante indicar que Mera y Sierra y Neira (2014) reportaron un $46,6 \%$ (34/73) de perros positivos al estudio serológico para $E$. canis, con cuadro clínico compatible con ehrlichiosis monocítica canina, aunque se desconoce la especie de garrapata implicada en estos casos.

Amblyomma tigrinum, la segunda especie en frecuencia reportada en este trabajo (Tabla 1), es una garrapata comúnmente asociada a carnívoros, sean silvestres o domésticos (Guglielmone et al., 2014) y ocasionalmente al hombre (Nava et al., 2006; Guglielmone et al., 2007). En América del Sur, se destaca por su capacidad para adaptarse a ambientes con características climáticas con- trastantes, lo que le confiere un alto potencial de distribución (Guglielmone et al., 2006). La baja frecuencia registrada de $A$. tigrinum (Tabla 1 ) puede deberse a que las formas inmaduras de esta especie utilizan a aves y roedores como hospedadores intermediarios (Guglielmone et al., 2006), por lo que su permanencia en zonas urbanas no sería estable, sugiriendo una mayor presencia en zonas peri urbanas y rurales (Guglielmone et al., 2006). Es de destacar que se observó una mayor presencia de ejemplares de $A$. tigrinum en los departamentos de Luján de Cuyo, Lavalle y Las Heras, lo que podría deberse a que amplios sectores de los ejidos urbanos de estos departamentos se encuentran cercanos a la zona de pedemonte o zonas rurales, pudiendo ser consideradas áreas periurbanas.

En cuanto al rol de A. tigrinum como vector de patógenos, esta especie ha sido hallada infectada con $R$. parkeri en Bolivia, Uruguay y Argentina (Tomassone et al., 2010; Lado et al., 2014; Romer et al., 2014), mientras que recientemente se ha descripto su infección con dos especies de Ehrlichia sin identificar en las provincias de San Luis y Córdoba (Cicuttin et al., 2017b).

En la región de Cuyo en general, y en Mendoza en particular, es poca la información con la que se cuenta respecto de los vectores establecidos, en particular las especies de garrapatas presentes. Por ello, los resultados del presente trabajo se consideran relevantes para un mejor conocimiento de las especies de garrapatas que parasitan caninos domésticos en zonas urbanas del Gran Mendoza. Ante esto, se considera de gran relevancia la necesidad de profundizar los estudios en la región de Cuyo. Ante los sugerentes hallazgos clínicos y serológicos, es necesario verificar la presencia de $E$. canis mediante técnicas de mayor fiabilidad (por ej., técnicas moleculares), al tiempo que identificar correctamente el linaje de los ejemplares de Rh. Sanguineus s.l. presentes en los perros infectados. Aún más importante debido a su rol en la transmisión de patógenos al hombre, deben continuarse los estudios acerca de la presencia, distribución y estado de infección de la especie A. tigrinum. Es por todo esto que la vigilancia vectorial debe ser, sin duda, una de las ramas a potenciar, fortalecer y difundir entre la comunidad sanitaria, para establecer sistemas de detección tempranos que permitan a los agentes sanitarios anticipar posibles episodios epidémicos, ya sean de tipo local o global.

\section{Agradecimientos}

Especial agradecimiento a la Dra. Evelina Tarragona (INTA Rafaela) por la revisión crítica de este manuscrito. 


\section{Bibliografía}

Cicuttin GL. 2014. Capítulo Rickettsiosis urbanas: Rickettsia massiliae. Libro "Temas de Zoonosis VI" Asociación Argentina de Zoonosis. pp. 281-286.

Cicuttin GL, Tarragona EL, De Salvo MN, Mangold AJ, Nava S. 2015. Infection with Ehrlichia canis and Anaplasma platys (Rickettsiales: Anaplasmataceae) in two lineages of Rhipicephalus sanguineus sensu lato (Acari: Ixodidae) from Argentina. Ticks Tick Borne Dis.6: 724-29.

Cicuttin GL, De Salvo MN, Silva D, Brito M, Nava S. 2017a. Ehrlichia canis (Rickettsiales: Anaplasmataceae) en garrapatas Rhipicephalus sanguineus sensu lato del linaje templado (Acari Ixodidae), provincia de Buenos Aires, Argentina. Revista FAVE sección Ciencias Veterinarias 16: 93-96.

Cicuttin GL, De Salvo MN, Nava S. 2017b. Two novel Ehrlichia strains detected in Amblyomma tigrinum ticks associated to dogs in peri-urban areas of Argentina. Comp. Immunol. Microbiol. Infect. Dis. 53: 40-44.

Dantas-Torres F. 2010. Biology and ecology of the brown dog tick, Rhipicephalus sanguineus. Parasit. Vectors 3: 26

Debárbora VN, Oscherov EB, Guglielmone AA, Nava S. 2011 Garrapatas (Acari: Ixodidae) asociadas a perros en diferentes ambientes de la provincia de Corrientes, Argentina. InVet 13: 4551.

Eiras DF, Basabe J, Scodellaro CF, Banach DB, Matos ML, Krime A, Baneth G. 2007. First molecular characterization of canine hepatozoonosis in Argentina: evaluation of asymptomatic Hepatozoon canis infection in dogs from Buenos Aires. Vet. Parasitol. 149: 275-279.

Eiras DF, Basabe J, Mesplet M, Schnittger L. 2008. First molecular characterization of Babesia vogeli in two naturally infected dogs of Buenos Aires, Argentina. Vet. Parasitol. 157: 294-298.

Guglielmone AA, Estrada-Peña A, Keirans AJ, Robbins RG. 2004 Las garrapatas (Acari. Ixodida) de la región zoogeográfica neotropical. Instituto Nacional de Tecnología Agropecuaria. Buenos Aires, Argentina. 142 pp.

Guglielmone AA, Beati L, Barros-Battesti DM, Labruna MB, Nava S, Venzal JM, Estrada-Peña A. 2006. Ticks (Ixodidae) on humans in South America. Exp. Appl. Acarol. 40: 83-100.

Guglielmone AA, Nava S, Mangold AJ, Caparros JA. 2007. Las garrapatas del norte de Córdoba, Argentina. Rev. Med. Vet. Buenos Aires. 88: 57-61

Guglielmone AA, Robbins RG, Apanaskevich DA, Petney TN Estarada-Peña A, Horak IG. 2014. The Hard Ticks of the World: (Acari: Ixodida: Ixodidae). Springer, Dordrecht. 738pp.

Informe del Instituto Nacional de Vitivinicultura. Departamento de Estadística y Estudios de Mercado. Subgerencia de Estadística y Asuntos Técnicos Internacionales. 2016. http://www.inv.gov.ar/inv contenidos/pdf/estadisticas/tespecial es/2016/INFORME AREA CENTRO.pdf
Labruna MB, Pacheco RC, Nava S, Brandao PE, Richtzenhain L, Guglielmone AA. 2007. Infection by Rickettsia bellii and Rickettsia amblyommii in Amblyomma neumanni ticks from Argentina. Microb. Ecol. 54: 126-133.

Lado P, Castro O, Labruna MB, Venzal JM. 2014. First molecular detection of Rickettsia parkeri in Amblyomma tigrinum and Amblyomma dubitatum ticks from Uruguay. Ticks Tick Borne Dis. 5: 660-662

Mera y Sierra R, Neira, G. 2014. Ocurrencia de Ehrlichia canis en caninos de la provincia de Mendoza. III Congreso Panamericano de Zoonosis/ V Congreso Argentino de Zoonosis Ciudad de La Plata, CD.

Monje LD, Linares MC, Beldomenico PM. 2016. Prevalence and infection intensity of Rickettsia massiliae in Rhipicephalus sanguineus sensu lato ticks from Mendoza, Argentina. Microbes Infect. 18: 701-705.

Moraes-Filho J, Marcili A, Nieri-Bastos FA, Richtzenhain LJ, Labruna MB. 2011. Genetic analysis of ticks belonging to the Rhipicephalus sanguineus group in Latin America. Acta Trop. 117: 51-5.

Moraes-Filho J, Krawczak FS, Costa FB, Soares JF, Labruna MB. 2015. Comparative evaluation of the vector competence of four south american populations of the Rhipicephalus sanguineus group for the bacterium Ehrlichia canis, the agent of canine monocytic ehrlichiosis. PLoS One. 10: e0139386.

Nava S, Caparros JA, Mangold AJ, Guglielmone, AA. 2006. Ticks (Acari: Ixodida: Argasidae, Ixodidae) infesting humans in Northwestern Córdoba Province, Argentina. Medicina, Buenos Aires 66: 225.

Nava S, Mastropaolo M, Venzal JM, Mangold AJ, Guglielmone AA. 2012. Mitochondrial DNA analysis of Rhipicephalus sanguineus sensu lato (Acari: Ixodidae) in the southern cone of South America. Vet. Parasitol. 190: 547-55.

Nava S, Venzal JM, González Acuña D, Martins TF, Guglielmone AA. 2017. Ticks of the Southern Cone of America. Ed. Academic Press, London. 375 pp.

Parola $\mathrm{P}$, Paddock CD, Scolovschi $C$, Labruna $M B$, Mediannokov O, Kernif T, Abdad M Y, Stenos J, Bitam I, Fournier PE, Raoult D. 2013. Update on tick-borne rickettsioses around the world: a geographic approach. Clin. Microbiol. Rev. 26:657-702.

Romer Y, Nava S, Govedic F, Cicuttin G, Denison AM, Singleton J, Kelly AJ, Kato Cl, Paddock CD. 2014. Rickettsia parkeri rickettsiosis in different ecological regions of Argentina and its association with Amblyomma tigrinum as a potential vector. Am. J. Trop. Med. Hyg. 91: 1156-1160

Tomassone L, Conte V, Parrilla G, De Meneghi D. 2010. Rickettsia infection in dogs and Rickettsia parkeri in Amblyomma tigrinum ticks, Cochabamba Department, Bolivia. Vector Borne Zoon. Dis. 10: 953-958.

Venzal JM, Estrada-Peña A, Portillo A, Mangold AJ, Castro O, Souza CG, Oteo JA. 2012. Rickettsia parkeri: a rickettsial pathogen transmitted by ticks in endemic areas for spotted fever rickettsiosis in southern Uruguay. Revista do Instituto de Medicina Tropical de Sao Paulo 54: 131-134. 
Walker JB, Keirans JE, Horak IG. 2000. The Genus Rhipicephalus (Acari: Ixodidae): A Guide to the Brown Ticks of the World. Ed. Cambridge University Press, Cambridge. 643 pp. 